\title{
La critique littéraire suisse: Autour de l'Ecole de Genève, "CEuvres et Critiques», XXVII, 2
}

\section{Cecilia Rizza}

\section{(2) OpenEdition}

1 Journals

\section{Edizione digitale}

URL: https://journals.openedition.org/studifrancesi/40202

DOI: $10.4000 /$ studifrancesi.40202

ISSN: 2421-5856

Editore

Rosenberg \& Sellier

\section{Edizione cartacea}

Data di pubblicazione: 1 juillet 2004

Paginazione: 145-146

ISSN: 0039-2944

\section{Notizia bibliografica digitale}

Cecilia Rizza, "La critique littéraire suisse: Autour de l'Ecole de Genève, «EFuvres et Critiques», XXVII, 2», Studi Francesi [Online], 142 (XLVIII | I) | 2004, online dal 30 novembre 2015, consultato il 09 septembre 2021. URL: http://journals.openedition.org/studifrancesi/40202 ; DOI: https://doi.org/10.4000/ studifrancesi.40202

Questo documento è stato generato automaticamente il 9 septembre 2021.

\section{(c)}

Studi Francesi è distribuita con Licenza Creative Commons Attribuzione - Non commerciale - Non opere derivate 4.0 Internazionale. 


\title{
La critique littéraire suisse: Autour de l'Ecole de Genève, "CEuvres et Critiques», XXVII, 2
}

\author{
Cecilia Rizza
}

\section{NOTIZIA}

La critique littéraire suisse: Autour de l'Ecole de Genève, «CEuvres et Critiques», XXVII, 2, 2002, pp. 329.

1 Rendendo omaggio in questo suo numero alla cosiddetta «scuola di Ginevra», la rivista creata e diretta da Wolfgang Leiner eccezionalmente rinuncia al suo scopo istituzionale di mettere a confronto i testi letterari con la storia della loro ricezione critica per soffermarsi invece sull'opera di quegli studiosi che, proprio grazie alla loro indipendenza da metodi e schemi prefissati, e pur conservando intatti i fondamenti filologici della ricerca, hanno favorito, nelle seconda metà del secolo scorso, il profondo rinnovamento della critica, specie per quanto riguardava la letteratura francese. Denominatore comune dell'attività di questi critici non è tanto, o non soltanto, l'aver esercitato la loro professione di Docenti nell'Università di Ginevra, ma il loro rifiuto del positivismo lansoniano, la scelta di una lettura dei testi nella loro interezza e specificità, l'attenzione alle altre espressioni della creazione artistica con le quali la letteratura è in stretta relazione e alle letterature di altri paesi europei, il rifiuto di una aprioristica e dogmatica scelta metodologica. Come già osservava Franco Simone nella presentazione al Supplemento di «Studi francesi» del 1968 dedicato alla Nouvelle critique in cui figuravano tra gli altri i contributi di George Poulet e di Jean Starobinski, (autori presenti insieme a Leo Spitzer, Marcel Raymond e Jean Rousset, nei primi numeri della rivista nei lontani anni Cinquanta), questi studiosi mettevano in crisi la vecchia concezione critica risalente al Sainte-Beuve, ancora dominante in Francia, specie presso 
i Sorbonnards, con il perdurante rito della tesi di dottorato e nonostante la spinta innovativa della critica per così dire militante.

2 A distanza di tanti anni, Michel Butor, nell'interessante intervista di Olivier Pot pubblicata nelle pagine finali di questo fascicolo, sottolinea, ancora una volta, l'estraneità dei critici dell'école de Genève dallo storicismo tradizionale che si riassume nella ben nota formula de l'homme et l'cuvre ed aggiunge, a ulteriore conferma della loro indipendenza, il loro rifiuto di tutte quelle forme di critica diventate alla moda nella seconda metà del Novecento che mutuano i metodi di ricerca da altre scienze, quali la psicanalisi, la sociologia, la linguistica strutturale, o si lasciano influenzare, più o meno coscientemente, dalle ideologie politiche. Gli studiosi ginevrini infatti non cercano d'imporre al testo un significato aprioristicamente determinato: «C'est le texte qui sert de pierre de touche pour les théories critiques qu'on lui confronte et c'est toujours le texte qui a raison. Les outils critiques extérieurs se révèlent plus ou moins féconds, mais si tel texte n'illustre pas bien telle thèse marxiste ou psychanalytique, ce doit être que la thèse marxiste ou psychanalytique était insuffisante» (p. 285).

Il fascicolo si apre con un'esauriente messa a punto critica di Olivier Pot e un importante contributo di J.-P. Richard che ricorda i suoi rapporti con gli amici ginevrini. Seguono alcuni articoli di A. Compagnon, M. Leymarie e H. Chudak su Albert Thibaudet e lo studio dedicato da S. Michaud ad Albert Béguin. Sia Thibaudet sia Béguin possono solo impropriamente essere considerati appartenenti alla scuola di Ginevra: critici molto diversi tra loro sono tuttavia entrambi buon conoscitori delle letterature e delle culture di altri paesi europei, soprattutto degli scrittori e dei critici tedeschi, e perciò particolarmente attenti alle problematiche filosofiche e sensibili ai valori etici e alle implicazioni morali dei testi letterari. Il costante riferimento alla storia delle idee si coniuga, nell'opera del Thibaudet con l'attenzione alle realtà formali riconoscibili e caratterizzanti i grandi autori , mentre nel Béguin meritano di essere sottolineati, al di là della sua opera più nota e discussa L'âme romantique et le rêve, i suoi rapporti con i più importanti poeti contemporanei, da Breton ad Aragon, a Saint-John Perse, a Eluard, come editore dei «Cahiers du Rhône». L'apertura «europea» di questi critici trova nuovo riscontro nell'opera di Denis de Rougemont come sottolinea opportunamente P. Dethourens.

4 L'analisi delle carte di Georges Poulet permette a S. Cudre-Mauroux di cogliere, nelle sue fasi principali, il metodo di lavoro di questo critico: scelta di un autore di cui viene letta integralmente l'opera, sottolineatura di quelle che si possono considerare le costanti tematiche e formali al fine d'individuare una rete di connessioni che valgano alla migliore comprensione del testo. Anche M. Dore utilizza delle note manoscritte per ricostruire la genesi della tesi di A. Viatte e definirne per tale via il metodo di ricerca.

5 La parte centrale e più importante del fascicolo è riservata, giustamente, ai veri, grandi maestri della scuola di Ginevra, non solo il già citato Poulet, ma soprattutto Marcel Raymond, Jean Starobinski e Jean Rousset e proprio alla memoria di quest'ultimo, recentemente scomparso, è dedicato questo numero della rivista. S'ingannerebbe tuttavia chi cercasse una sorta di dipendenza reciproca di questi studiosi nella loro attività di ricerca; non sempre le loro posizioni coincidono, come risulta dalla corrispondenza Poulet-Raymond analizzata da A. Raybaud. Comune è proprio la loro autonomia di giudizio che non si smentisce neppure nei reciproci rapporti personali: basti pensare al confronto Raymond-Rousset a proposito di Manierismo e Barocco. Per il primo forma e contenuto non possono essere disgiunti ma esistono nella loro stretta 
interdipendenza e come tali vanno studiati. Non ci si stupirà quindi se, come osserva il Ménager, il Ronsard di Marcel Raymond, cui era stato rimproverata una sorta di ritorno alla critica storica, non si esaurisce in una esemplare lettura unitaria di tutta l'opera del poeta della Pléiade, ma si apre alla scoperta e alla valorizzazione del Manierismo in letteratura, e conduce altresì alla discussione sul Barocco. Senza voler negare il debito del Rousset nei confronti di Marcel Raymond è certo che La littérature de l'âge baroque en France, mutuando dalle arti figurative suggestivi esempi, inaugura un'originale metodologia che comporta un'interpretazione radicalmente diversa della letteratura francese del XVII secolo. Che l'opera del Rousset sia apparsa agli studiosi del tempo, assai più rivoluzionaria di quella dei Raymond si evince anche dalla recensione di $\mathrm{A}$. Pizzorosso pubblicata nel 1954 sulla «Rivista di Letterature moderne e comparate» e qui riprodotta, in cui, pur riconoscendo al Rousset il merito di aver riscoperto e valorizzato autori ed opere fin allora poco o mal conosciuti, lo studioso italiano osservava come «Alcune questioni di metodo o di principio potrebbero essere discusse» dal momento che il Rousset «rifugge da ogni presa di posizione dogmatica» (p. 296). Rileggendo oggi quello che, giovane laureata, scrivevo recensendo su «Convivium» (n.s. 1954 n.3) il volume del Rousset, mi rendo conto di quanto anch'io, che di lì a poco avrei riconosciuto in Rousset un mio maitre en Baroquie, fossi stata molto prudente nel valutare l'importanza della sua opera.

6 Libertà e indipendenza anche da un maestro riconosciuto e stimato caratterizzano il rapporto Raymond-Starobinski come appare dal confronto che A. Tournon stabilisce sulla diversa lettura che i due critici propongono dell'opera di Montaigne e dall'analisi di M. Olender sulla del tutto originale démarche critique di Starobinski. Estremamente significativa risulta perciò l'intepretazione dell'opera di Baudelaire proposta dal Raymond e poi dallo Starobinski, l'uno e l'altro, sia pure in maniera diversa, come ben chiarisce $\mathrm{P}$. Labarthe, all'origine di tutta la critica più recente su questo poeta. In questa prospettiva acquista un particolare interesse non soltanto la ricezione che l'opera del Rousset ha ricevuto in Romania di cui ci parla I. Pop e che potrebbe essere completata da molti altri analoghi contributi concernenti l'Italia, la Germania, gli Stati Uniti, ecc., ma lo sviluppo che le sue ricerche sul Barocco hanno avuto presso critici come G. Mathieu-Castellani e Cl.-G. Dubois di cui gli articoli qui pubblicati rendono esplicita testimonianza. Non si dimentichi inoltre che lo stesso Rousset, a distanza di anni, consapevole degli approfondimenti che i suoi studi avevano sollecitato non ha esitato a ritornare sul problema del Barocco letterario in Francia rivedendo e modificando anche in modo radicale le sue precedenti posizioni.

7 Veri maestri, è questa la lezione che si ricava dalla lettura dei contributi raccolti in questo eccellente fascicolo, sono certamente coloro che con grande coerenza ed onestà intellettuale aprono nuove prospettive alla ricerca, senza arroccarsi su posizioni dogmatiche e allo stesso tempo suscitano allievi che, pur richiamandosi al loro insegnamento, sanno distaccarsene per elaborare, a loro volta, nuove metodologie e scoprire nuovi orizzonti.

8 Dobbiamo essere grati ai responsabili scientifici e al Direttore di «Euvres et critiques» per la particolare attenzione dedicata alla critica letteraria svizzera che fa capo alla «scuola di Ginevra», una iniziativa che non soltanto ci permette di ripercorrere mezzo secolo di studi fortemente innovativi e forieri di nuove ricerche, ma ricorda, a noi tutti, il ruolo di ogni studioso e il suo obbligo morale di saper essere o almeno tentare di essere, in qualche misura, anche un Maestro. 